\title{
Calibration of electromagnetic tracking devices
}

\author{
Volodymyr Kindratenko \\ National Center for Supercomputing Applications (NCSA) \\ University of Illinois at Urbana-Champaign (UIUC) \\ 405 N. Mathews Avenue, Urbana, IL 61801, USA
}

\begin{abstract}
Electromagnetic tracking devices are often used to track location and orientation of a user in a virtual reality environment. Their precision, however, is not always high enough due to the dependence of the system on the local electromagnetic field that can be easily altered by many external factors. The purpose of this article is to give an overview of the calibration techniques used to improve the precision of the electromagnetic tracking devices and to present a new method that compensates both the position and orientation errors. It is shown numerically that significant improvements in the precision of the detected position and orientation can be achieved with a small number of calibration measurements to be taken. Unresolved problems and research topics related to the proposed method are discussed.
\end{abstract}

\section{Keywords}

Electromagnetic Tracker, Tracker Calibration, Polynomial Fit, Virtual Reality

\section{Introduction}

Virtual reality (VR) systems often rely on six-degree-of-freedom electromagnetic trackers to determine position and orientation of a user in the working space. Among most frequently used long-range systems of this type are Flock of Birds (Ascension Technology Corporation) and 3Space Fastrak (Polhemus, Inc.). Both devices are based on a design that uses orthogonal electromagnetic fields to sense 3D position and orientation [1-4]: the electromagnetic transmitter contains three orthogonal coils that are pulsed in a sequence, the receiver also has three orthogonal coils that measure the electromagnetic field produced by the transmitter, the strength of the received signals is compared to the strength of the sent pulses to determine the position and compared to each other to determine the orientation. The measurements are rather noisy, therefore an additional filtering is required. Working range of both systems is clamed to be up to 10 feet from the transmitter, but the accuracy of the systems decreases as the distance between the transmitter and the receiver increases. Also, due to the dependence of the measurements on the local electromagnetic field, the tracking systems are sensitive to the 
ambient electromagnetic environment. If there is either a metal, or other conductive materials, or equipment that produces an electromagnetic field near the tracker's transmitter or receiver, the transmitter signals are distorted and the resulting measurements contain both static and dynamic error. An experimental investigation was undertaken [2] to study the effects of external fields present in a typical working environment and the presence of metals near the transmitter or receiver. Static errors as high as 4 feet have been observed near the maximum range of the tracking system [3]. The manufacturers of the tracking systems suggest that there should be no metal components near the transmitter and receiver, which is often not possible to achieve due to the construction limitations of the VR systems.

On the other hand, it has been shown [3-5] that the quality of VR experience and user perception and performance is quite dependent on the accuracy of the tracking system. K. Meyer et al. [4] discussed the effects of inaccurate position tracking on the illusion of presence in a virtual environment and pointed out a number of problems that may result from it including motion sickness, absence of perceptual adaptation, intersensory conflicts, etc. In fact, the entire system usability can be compromised because of a low accuracy of tracking. This is especially true for the augmented reality applications where the objects from both the virtual and real world are present in the same environment. For example, when there is a misalignment between the user's hand and its graphical representation, the illusion of reality tends to break down because of the intersensory conflict: felt and observed hands are not the same.

One way to overcome these problems is to increase the accuracy of tracking by compensating the measurements for the errors through experimentally established dependencies between the actual receiver position/orientation and that reported by the tracking system. This procedure is called tracker calibration. Assuming that the transmitter's position is fixed and the surrounding metal does not move, the static error is a function of the position of the receiver and it can be corrected as long as the magnetic field does not "fold back" on itself [1]. In practice, typically only the position is corrected. Usually it is done applying a trilinear interpolation between the distorted measurements and their known in advance true values at some points. Such a data set is called calibration table. The use of this technique is based on the assumption that the distortion is linear within a given subspace, which is not always the case. Also, to make this working, a lot of precise measurements are required. For example, in the case of the CAVE (Cave Automated Virtual Environment) VR system [6], which is 10x10x10 feet cube, 1000 measurements are required to obtain a full 1 foot interval calibration table. Since, in practice not all the areas are reachable by the user, it was found that about 400 measurements are enough to calibrate the CAVE [3]. Still, this is inappropriate for the systems where frequent modifications of the environment occur. For example, an installation of a vehicle mockup platform in the CAVE, as required for the application described in [7], results in severe distortions to the tracker measurements that cannot be compensated by the same calibration table as the environment without the platform. Therefore, for each addition to or modification of the environment a new calibration table (new set of measurements) has to be created.

Because of the absence of a calibration technique that is simple to setup and compensates both for position and orientation errors, there is no good calibration software available. Up to the author's knowledge, only the CAVE library (VRCO) has a 
build-in trackers calibration support. However, because of the specific way the measurements have to be taken [3], this functionality of the software is seldom used. Both Ascension Technology Corporation and Polhemus, Inc. provide some software to assist in the calibration process, but it is not developed into a finished product.

The purpose of this paper is to give an overview of existing calibration methods reported in the literature and to present a new calibration technique that corrects both the positional and rotational errors in an electromagnetic tracking system and requires a relatively small number of measurements to be taken. An implementation of the method in a form of a software library that can be linked with a third-party code is available.

\section{Related work}

The problem of the electromagnetic trackers calibration has been given a constant attention in the literature. F. Raab et al. [1] suggested that corrections of the distorted position measurements can take the form of additive vectors or a sequence of rotations, and can be stored in either a look-up table or as polynomials in the position parameters. Much of the work done after [1] implements a variation of one of these two methods. Table 1 summarizes all the methods described below.

S. Bryson [8] published results of his work on the calibration of static distortion of position performed at the NASA Ames VIEW Lab. The floor of the lab was labeled with distance marks and a pegboard on a stand was built to place the Isotrack (Polhemus, Inc.) tracker sensor at known positions and fixed orientation. A regular 8x8x6 feet volume was measured with the 12 inches step between the measurement sights in order to build a calibration table and with the same step plus 6 inches offset in order to compose a validation table. At each sight, 60 measurements were taken and the average and standard deviation were computed. Preliminarily study of the data suggested that the calibration would not work beyond 50 inches from the transmitter due to the tracker error, noise, and the repeatability problems. Two calibration methods, polynomial calibration and weighted table lookup, were applied. Polynomials of the order 1 through 8 for each position component $x, y$, and $z$ were computed from the calibration table via the least-square fit method and tested on the data from the validation table. It was found via the correlation analysis that the polynomials of the $4^{\text {th }}$ order were the most suited. The lookup table method was applied in the following way: for a given measured position the nearest pre-measured position in the calibration table was located and the corresponding actual position was interpolated using several nearby points in the table. Two interpolation schemes ware evaluated: the linear lookup calibration in which each weight was defined as a linear function of distance, and the bump lookup calibration in which each weight was defined as an exponentially decaying function of distance. The results showed that the $4^{\text {th }}$ order polynomial calibration reduces the error from 4 inches to 2 inches up to the distance of 40 inches form the source and produced the smallest error vectors overall, but had noticeably large error vectors near the source. The bump lookup method provided the smallest error vectors only in the neighborhood of the source, and the linear lookup introduced considerable amounts of error and scatter.

M. Ghazisaedy et al. [3] published results of the work on the calibration of the electromagnetic trackers performed at the Electronic Visualization Laboratory at the University of Illinois at Chicago. A custom-built ultrasonic measuring device (UMD) 
was used to obtain a precise Extended Range Transmitter Flock of Birds (Ascension Technology Corporation) magnetic tracker receiver location in the CAVE. The UMD consisted of 4 ultrasonic transducers, one to measure the distance to each wall and floor of the CAVE, generating an ultrasonic sound signals and sending them towards the walls. The sound was reflected from the walls and detected by the transducers, distances were obtained by measuring the time interval between the moment the sound is transmitted and the echo is received. The overall error of the UMD was less than 1.8 inches in the 10 feet CAVE. The Flock of Birds sensor was attached atop the UMD. The CAVE was filled by a 3D stereo graphics images of 1-inch boxes on 1-foot intervals, 400 in total, and a 1-inch cursor showed the position of the sensor; a person wearing 3D glasses held the UMD reasonably straight and moved it until the virtual cursor visited all the displayed boxes. A calibration table was built by recording the positions measured by the electromagnetic tracking system and the UMD at each displayed box, a validation table was obtained by collecting data at one foot intervals on half-foot centers. Trilinear interpolation was used to correct the Flock of Birds readings. The maximum error before the calibration was seen to be 4 (0.6) feet over 10 (3) feet range, the error after the calibration was 0.27 (0.13) feet in the same 10 (3) feet range. Clearly, the procedure performed better correcting larger errors than smaller ones. The authors concluded that the improvements produced by the calibration were very much worth the effort.

M. Czernuszenko et al. [9] developed a line-of-sight method for correcting static errors in the position component of electromagnetic tracking. The basic idea behind the method is to build a correction table based on the misalignment between the physical objects at known locations and drawn by the graphics system similar objects in the same locations as the real ones. If there are no errors in the reported sensor position, the displayed objects would be superimposed on the physical ones when viewed from any location, otherwise they would not coincide. The user moved the drawn image until it aligned with the real object. The amount and direction of the movement gave a correction vector that was used to update each point in the calibration table according to an equation that adds the weighted correction vector to all the points in the table. The user moved in the environment and made additional corrections where they seemed to be necessary; each time a new correction vector was introduced, the calibration table was recalculated. The method was tested in the CAVE where three physical targets were suspended 5 feet above the floor, 2 feet apart, 20 inches from the front wall. After about 25 corrections, the drawn targets seemed to be always superimposed on the real ones and all the following corrections were smaller than 0.2 feet.

S. Ellis et al. [5] developed a technique that compensated for both position and rotation distortions. The goal of the work was to study sensor spatial distortion, visual latency, and update rate effects on human performance in a virtual environment, therefore it required a well-calibrated tracking system. A calibration table was build over the $72 \times 48 \times 30$ inches volume with the 6 inches distance between the nodes. A validation table was build by taking the measurements at intermediate locations. An adaptation of a point location technique based on tetrahedral elements [10] was used for the position correction. While the trilinear interpolation cannot be easily inverted to provide the mapping between the measured and true coordinates using the calibration table, the technique based on tetrahedral elements allows to evaluate true coordinates directly from the measured ones. The method reduced the residual of the position errors for the data 
from the validation table approximately by sevenfold. Errors in orientation were measured by the quaterions that would rotate the measured local verticals, approximately surface normals to each quadrilateral patch, into true vertical. The orientation errors within each calibrated cell were corrected by inverse rotations based on the error measurements at adjacent calibration grid nodes. No results were published on the quality of the rotation calibration.

Results from [3, 5, 8, 9] suggest the following:

1. All the methods require some form of a calibration table. Usually such a table is obtained measuring tracker position at known true locations. Only the method of Ghazisaedy et al. [3] requires measuring true location at given tracked positions.

2. Because of the assumption that the magnetic field is distorted linearly within a given sub-volume, interpolation-based techniques work well when a lot of equivalently spaced measurements are available.

3. Fit-based techniques generally perform better than interpolation-based ones. Since the fit can take a form of a high-order polynomial that may very well capture the shape of the distorted magnetic field, fewer measurements may be needed.

4. Orientation calibration typically is not performed. No good orientation calibration methods have been proposed.

\section{The method}

In the present method, high-order polynomials are used for the position correction. The orientation correction also employs high-order polynomials with an additional rotation correction afterwards.

\section{Position correction}

Distorted position $\left(x_{i}, y_{i}, z_{i}\right)$ and orientation $\left(\alpha_{i}, \beta_{i}, \gamma_{i}\right)$ (azimuth, elevation, and roll), measurements are collected at $m$ points of known true location $\left(\bar{x}_{i}, \bar{y}_{i}, \bar{z}_{i}\right)$ and known true orientation $\left(\bar{\alpha}_{i}, \bar{\beta}_{i}, \bar{\gamma}_{i}\right)=(0,0,0)$ of the receiver at each point $i$ and stored in a calibration file.

Position correction equations can be generated by fitting a curve or a surface to the data points. In the case of a polynomial fit of the order $r$, these equations take the general form of

$$
\tilde{x}=x+\sum_{j=1}^{n} c_{x j} x^{p_{j}^{\prime}} y^{p_{j}^{\prime \prime}} z^{p_{j}^{\prime \prime}}, \tilde{y}=y+\sum_{j=1}^{n} c_{y j} x^{p_{j}^{\prime}} y^{p_{j}^{\prime \prime}} z^{p_{j}^{\prime \prime}}, \widetilde{z}=z+\sum_{j=1}^{n} c_{z j} x^{p_{j}^{\prime}} y^{p_{j}^{\prime \prime}} z^{p_{j}^{\prime \prime}}
$$

where $c_{x j}, c_{y j}$, and $c_{z j}$ are polynomial coefficients; $n$ is the number of terms in the fitting polynomial that depends on the order $r$ of the polynomial and is equal to the number of permutations $\left\{p_{j}^{\prime}, p_{j}^{\prime \prime}, p_{j}^{\prime \prime \prime}\right\}$ where $p_{j}^{\prime}, p_{j}^{\prime \prime}$ and $p_{j}^{\prime \prime \prime}$ are the powers of the corresponding terms such that $\left.i) p_{j}^{\prime}, p_{j}^{\prime \prime}, p_{j}^{\prime \prime \prime} \in\{0,1, \ldots, r\}, i i\right) \quad p_{j}^{\prime}+p_{j}^{\prime \prime}+p_{j}^{\prime \prime \prime} \leq r$ for any $j=1, \ldots, n$, and iii) all permutations $\left\{p_{j}^{\prime}, p_{j}^{\prime \prime}, p_{j}^{\prime \prime \prime}\right\}$ are unique; $\widetilde{x}, \tilde{y}$, and $\widetilde{z}$ are corrected values of the distorted measurements $x, y$, and $z$. For example, the second-order polynomial for $x$ coordinate looks like this:

$$
\tilde{x}=x+\left(c_{x 1} x^{2}+c_{x 2} y^{2}+c_{x 3} z^{2}+c_{x 4} x y+c_{x 5} x z+c_{x 6} y z+c_{x 7} x+c_{x 8} y+c_{x 9} z+c_{x 10}\right)
$$


where the number of terms in the polynomial $n=10$, powers $p_{1}^{\prime}=2, p_{1}^{\prime \prime}=0, p_{1}^{\prime \prime \prime}=0$, and so on.

In order to build the fitting polynomials, the polynomial coefficients $c_{x j}, c_{y j}$, and $c_{z j}$ must be computed. It can be done by solving the following simultaneous equations built for each coordinate $x, y$, and $z$ (all the equations below are given for $x$ coordinate only, equations for two other coordinates are similar):

$$
\left\{\begin{array}{c}
c_{x 1} x_{1}^{p_{1}^{\prime}} y_{1}^{p_{1}^{\prime \prime}} z_{1}^{p_{1}^{\prime \prime}}+\ldots+c_{x j} x_{1}^{p_{j}^{\prime}} y_{1}^{p_{j}^{\prime \prime}} z_{1}^{p_{j}^{\prime \prime}}+\ldots+c_{x n} x_{1}^{p_{n}^{\prime}} y_{1}^{p_{n}^{\prime \prime}} z_{1}^{p_{n}^{\prime \prime \prime}}=\bar{x}_{1}-x_{1} \\
c_{x 1} x_{i}^{p_{1}^{\prime}} y_{i}^{p_{1}^{\prime \prime}} z_{i}^{p_{1}^{\prime \prime}}+\ldots+c_{x j} x_{i}^{p_{j}^{\prime}} y_{i}^{p_{j}^{\prime \prime}} z_{i}^{p_{j}^{\prime \prime}}+\ldots+c_{x n} x_{i}^{p_{n}^{\prime}} y_{i}^{p_{n}^{\prime \prime}} z_{i}^{p_{n}^{\prime \prime \prime}}=\bar{x}_{i}-x_{i} \\
\ldots \\
c_{x 1} x_{m}^{p_{1}^{\prime}} y_{m}^{p_{1}^{\prime \prime}} z_{m}^{p_{1}^{\prime \prime}}+\ldots+c_{x j} x_{m}^{p_{j}^{\prime}} y_{m}^{p_{j}^{\prime \prime}} z_{m}^{p_{j}^{\prime \prime}}+\ldots+c_{x n} x_{m}^{p_{n}^{\prime}} y_{m}^{p_{n}^{\prime \prime}} z_{m}^{p_{n}^{\prime \prime}}=\bar{x}_{m}-x_{m}
\end{array}\right.
$$

that can be written in the matrix form $\mathbf{A t}=\mathbf{b}$ as

$$
\left(\begin{array}{ccccc}
a_{1,1} & \ldots & a_{1, j} & \ldots & a_{1, n} \\
: & : & : & : & : \\
a_{i, 1} & \ldots & a_{i, j} & \ldots & a_{i, n} \\
: & : & : & : & : \\
a_{m, 1} & \ldots & a_{m, j} & \ldots & a_{m, n}
\end{array}\right)\left(\begin{array}{c}
t_{1} \\
\ldots \\
t_{j} \\
\ldots \\
t_{n}
\end{array}\right)=\left(\begin{array}{c}
b_{1} \\
\ldots \\
b_{i} \\
\ldots \\
b_{m}
\end{array}\right)
$$

where $a_{i, j}=x_{i}^{p_{j}^{\prime}} y_{i}^{p_{j}^{\prime \prime}} z_{i}^{p_{j}^{\prime \prime}}, t_{j}=c_{x j}$, and $b_{i}=\bar{x}_{i}-x_{i}$. The type of solution these simultaneous equations may have is solely determined by the ranks of the matrix $\mathbf{A}$ and the augmented matrix $\mathbf{A} \mid \mathbf{b}$ : if $\operatorname{rank}(\mathbf{A}) \neq \operatorname{rank}(\mathbf{A} \mid \mathbf{b})$, the system has no solution, if $\operatorname{rank}(\mathbf{A})=\operatorname{rank}(\mathbf{A} \mid \mathbf{b})=n$, the system has a trivial solution; if $\operatorname{rank}(\mathbf{A})=\operatorname{rank}(\mathbf{A} \mid \mathbf{b})<n$, the system has many solutions.

Simultaneous equations can be solved numerically using so-called Gaussian elimination method. The idea behind the method is to transform the augmented matrix $\mathbf{A} \mid \mathbf{b}$ into an equivalent triangular matrix applying equivalent transformations such as $i$ ) moving the rows of the matrix, $i$ ) multiplying them by a constant that is not equal to zero, and iii) adding rows to each other. Once a triangular matrix is obtained, the solution of the system can be computed applying back substitution.

Typically, the number of equations, which is equal to the number of measurements $m$, is much higher than the number of terms $n$ in the polynomial. Taking into account the fact that the magnetic field distortion is typically non-linear, the solution of the resulting simultaneous equations typically does not exist. That is why we can only fit the surface to the data points (e.g., in the least square sense) instead of computing the exact solution. The idea is to find a set of polynomial coefficients $\hat{c}_{x j}$ such that polynomial

$$
\hat{c}_{x 1} x^{p_{1}^{\prime}} y^{p_{1}^{\prime \prime}} z^{p_{1}^{\prime \prime}}+\ldots+\hat{c}_{x j} x^{p_{j}^{\prime}} y^{p_{j}^{\prime \prime}} z^{p_{j}^{\prime \prime}}+\ldots+\hat{c}_{x n} x^{p_{n}^{\prime}} y^{p_{n}^{\prime \prime}} z^{p_{n}^{\prime \prime}}
$$

fits the error $\bar{x}-x$ in the least square sense. Coefficients $\hat{c}_{x j}$ can be computed by solving simultaneous normal equations build from the known true and measured values: 


$$
\left\{\begin{array}{c}
\sum_{j=1}^{n} \hat{c}_{x j} \sum_{i=1}^{m} a_{i, j} a_{i, 1}=\sum_{i=1}^{m} b_{i} a_{i, 1} \\
\sum_{j=1}^{n} \hat{c}_{x j} \sum_{i=1}^{m} a_{i, j} a_{i, k}=\sum_{i=1}^{m} b_{i} a_{i, k} \\
\ldots \\
\sum_{j=1}^{n} \hat{c}_{x j} \sum_{i=1}^{m} a_{i, j} a_{i, n}=\sum_{i=1}^{m} b_{i} a_{i, n}
\end{array}\right.
$$

where $k=1, \ldots, n$. Once polynomial coefficients $\hat{c}_{x j}$ are known, they can be used to compute the corrected value $\tilde{x}$ of $x$ coordinate:

$$
\tilde{x}=x+\sum_{j=1}^{n} \hat{c}_{x j} x^{p_{j}^{\prime}} y^{p_{j}^{\prime \prime}} z^{p_{j}^{\prime \prime \prime}}
$$

Correction equations for two other coordinates can be build in the same way using $y_{i}$, $\bar{y}_{i}, z_{i}$, and $\bar{z}_{i}$.

\section{Orientation correction}

Unfortunately, the above-described approach cannot be directly applied to correct orientation distortions. It must be modified to take into account the nature of the Euler angles that cannot be simply added together, rather they must be rotated.

Since the measurements were performed in such a way that $\left(\bar{\alpha}_{i}, \bar{\beta}_{i}, \bar{\gamma}_{i}\right)=(0,0,0)$ for $\forall i$, we can build and use fitting polynomials in the form

$$
\alpha^{*}=\sum_{j=1}^{n} \hat{c}_{\alpha j} x^{p_{j}^{\prime}} y^{p_{j}^{\prime \prime}} z^{p_{j}^{\prime \prime}}, \beta^{*}=\sum_{j=1}^{n} \hat{c}_{\beta j} x^{p_{j}^{\prime}} y^{p_{j}^{\prime \prime}} z^{p_{j}^{\prime \prime}}, \gamma^{*}=\sum_{j=1}^{n} \hat{c}_{y^{\prime}} x^{p_{j}^{\prime}} y^{p_{j}^{\prime \prime}} z^{p_{j}^{\prime \prime}}
$$

that fit the errors $\alpha^{*}, \beta^{*}$, and $\gamma^{*}$ instead of fitting the actual values of the Euler angles. Their coefficients $\hat{c}_{\alpha j}, \hat{c}_{\beta j}$, and $\hat{c}_{y j}$ can be computed in the same way as those in the case of $x, y$, and $z$.

Once the errors of $\alpha^{*}, \beta^{*}$, and $\gamma^{*}$ at a given measured location $(x, y, z)$ are computed, the corrected values $\widetilde{\alpha}, \widetilde{\beta}$, and $\widetilde{\gamma}$ can be obtained rotating $(\alpha, \beta, \gamma)$ by $\left(\alpha^{*}, \beta^{*}, \gamma^{*}\right)$. A correction rotation matrix is formed from $\left(\alpha^{*}, \beta^{*}, \gamma^{*}\right)$ :

$$
\mathbf{M}^{*}=\left(\begin{array}{ccc}
\cos \beta^{*} \cos \alpha^{*} & \cos \beta^{*} \sin \alpha^{*} & -\sin \beta^{*} \\
\sin \gamma^{*} \sin \beta^{*} \cos \alpha^{*}- & \cos \gamma^{*} \cos \alpha^{*}+ & \\
-\cos \gamma^{*} \sin \alpha^{*} & +\sin \gamma^{*} \sin \beta^{*} \sin \alpha^{*} & \sin \gamma^{*} \cos \beta^{*} \\
\sin \gamma^{*} \sin \alpha^{*}+ & \cos \gamma^{*} \sin \beta^{*} \sin \alpha^{*}- & \\
+\cos \gamma^{*} \sin \beta^{*} \cos \alpha^{*} & -\sin \gamma^{*} \cos \alpha^{*} & \cos \gamma^{*} \cos \beta^{*}
\end{array}\right)
$$


A measurement rotation matrix $\mathbf{M}$ is formed in the same way using $(\alpha, \beta, \gamma)$ and is multiplied by the correction rotation matrix $\mathbf{M}^{*}$. The resulting matrix $\widetilde{\mathbf{M}}=\mathbf{M} \mathbf{M}^{*}$ is then used to compute corrected Euler angles $\widetilde{\alpha}, \widetilde{\beta}$, and $\widetilde{\gamma}$ :

$$
\widetilde{\alpha}=\arctan \frac{\widetilde{\mathbf{M}}_{1,2}}{\widetilde{\mathbf{M}}_{1,1}}, \widetilde{\beta}=-\arcsin \widetilde{\mathbf{M}}_{1,3}, \widetilde{\gamma}=\arctan \frac{\widetilde{\mathbf{M}}_{2,3}}{\widetilde{\mathbf{M}}_{3,3}}
$$

\section{Results and discussion}

The technique was implemented in the form of a software library and tested with a Flock of Birds tracking system installed in the CAVE at the National Center for Supercomputing Applications at the University of Illinois at Urbana-Champaign. All the measurements were recorded in the CAVE coordinate system the origin of which is in the middle of the floor 5 feet away from the left, right, and front walls, $x$ axis points towards the right wall, $y$ axis points up, and $z$ axis points away from the front wall. Distances were measured in feet, angles were measured in degrees, but internally they were converted to radians. Transmitter's location in the CAVE coordinate system is $(0.06,8.42,-2.32)$. The floor of the CAVE was marked by two sets of perpendicular 1foot spaced lines. A simple sensor holder was designed consisting from a $1 \times 1 \times 0.1$ feet wooden platform with a housing attached at the top and a set of plastic pipes of the length $2,4,6$, and 8 feet that can be plugged into the housing. Moving the platform between the marks on the floor and changing the pipes allowed to place the sensor at points whose locations are known. After a very careful alignment, the precision of this measuring technique is believed to be \pm 0.5 centimeter, \pm 1 degree.

A calibration table was build by placing the receiver at 100 points of known location and collecting the values reported by the tracking system. Initially the receiver was placed at the point $(-4,2,-4)$, which is located near the left-down corner of the CAVE, and then was moved on the 2 feet grid until the entire CAVE space was covered. Later some of the points were excluded from the calibration file because the reported position values were out of workable range of the transmitter. The resulting calibration table contained 88 points. A validation table was acquired by measuring 64 points 5 feet from the floor, 1 foot apart starting at the point $(-3.49,5,-3.5)$.

Fig. 1 shows a surface built over the points from the validation table. Right edge of the surface is close to the front wall of the CAVE and corresponds to the measurements taken approximately 3.5 feet from the center of the CAVE; left edge corresponds to the measurements taken about 3.5 feet back from the center of the CAVE. As seen from the picture, there are minor distortions of the position measurements near the Flock of Birds' transmitter (right part of the surface is almost flat) and there are significant distortions observed for the measurements taken at 1.5, 2.5, and 3.5 feet back from the center of the CAVE (left part of the surface folds up). The largest distance between the true coordinates of the sensor and those reported by the tracking system is 1.56 feet, the largest angular distortion in 13 degrees is observed for the elevation.

Polynomial coefficients were built from the calibration table for the polynomials of the order 2,3,4, and 5. Measurements from the validation table were fitted by the derived polynomials and the resulting corrected values of the position and orientation 
were used to calculate some statistics regarding the quality of the fit. Table 2 summarizes the results. Average, standard deviation, min, and max were computed for the absolute values of the differences between true values of $x, y, z, \alpha, \beta$, and $\gamma$ and those reported by the tracker (rows $\Delta|x|, \Delta|y|, \Delta|z|, \Delta|\alpha|, \Delta|\beta|$, and $\Delta|\gamma|$ ) and for distances between the points defined by the true values of $x, y$ and $z$ and those reported by the tracker (row $\Delta|d|$ ). The same computations were performed after fitting the data by the polynomials of the $2^{\text {nd }}, 3^{\text {rd }}, 4^{\text {th }}$, and $5^{\text {th }}$ order. As seen from the table, polynomials of different order perform differently on different variables. For example, the smallest average of the residual for $y$ was obtained while fitting it by the $3^{\text {rd }}$ order polynomial whereas the $4^{\text {th }}$ order polynomial performed better for $z$. Overall, the $3^{\text {rd }}$ order polynomials performed well for all variables.

Fig. 2 contains measured (a) and fitted by the $3^{\text {rd }}$ order polynomial (b) values of $x$ coordinate of the points from the validation table plotted against its true value. As seen from the plots, fitted values are much closer to the true values (ideally all the dots should be on the dotted line) comparing to the non-fitted values. Fig. 3 presents frequency histograms obtained for the distances between the points defined by the true values of $x$, $y$ and $z$ and those reported by the tracker (a) and between the points defined by the true values of $x, y$ and $z$ and fitted by the $3^{\text {rd }}$ order polynomial (b). As seen from the histograms, only about $38 \%$ of the non-fitted points are within 0.26 feet from the true points. At the same time, about $75 \%$ of the fitted points are within the same distance of 0.26 feet from the true points. Fig. 4 shows improvements achieved while correcting azimuth with the $3^{\text {rd }}$ order polynomial. Measured and fitted values of the azimuth of all 64 points from the validation table are plotted. True value of azimuth for all the points is zero. In a few cases fitted values are larger than the measured values, but overall they are much closer to the true value, especially for the points 2.5-3.5 feet back from the center of the CAVE.

Finally, Fig. 5 shows a surface plotted over the points from the validation table after fitting them by the $3^{\text {rd }}$ order polynomial. The surface is much flatter comparing to Fig. 1, large distortions near the back of the CAVE are removed. However, the central part of the surface exhibits some distortions that contribute to the lover quality of the fit for the measurements taken in that area. This is in the agreement with the results obtained by S. Bryson [8] and points out a potential weakness of this approach. The largest distance between the true coordinates of the sensor and those fitted by the polynomials is 0.39 feet instead of 1.56 feet before fitting, the largest angular distortion for the elevation is 4 degrees instead of 13 degrees before the fitting.

How does all these relate to what a user experiences? Consider the system before the calibration. When a user moves back from the center of the CAVE, the entire scene moves down and tilts a bit depending on how far the user is back and left or right from the transmitter. This happens because in the tracked space the user's movement is not on the flat plane, rather it is on the curved surface similar to one shown in Fig. 1. This helps users to fill uncomfortable enough so they seldom go farther than a couple of feet from the center of the CAVE. However, the bigger issue is with the user's hand. In our setup we use a wand and often attach a virtual hand or pointer to it. When a position error is present in the system, the virtual hand is displaced from the physical one; when the error is changing with the movement, the virtual hand drifts all the time making it very difficult 
to perform any fine tasks. In one of our applications, user calls a virtual menu that appears in front of his body. If the user stays far enough from the transmitter, the distance between his actual hand and its virtual representation can be significant and rapidly changing as the hand moves. This makes the selection of menu items very difficult, sometimes hardly possible et all. However, with the calibration, this problem disappears. Also, as the user moves farther from the center of the CAVE, there is no scene deformation observed.

\section{Conclusions}

One of the goals of the study was to identify a technique that does not require too many measurements to be taken. In the above-discussed case, only 88 measurements were used resulting in as much as 4 times improved position and 3 times improved orientation detection precision. This may not look very impressive, but it could not be achieved applying a technique based on the trilinear interpolation with the same amount of the calibration points because of the assumption of the linearity of the magnetic field distortion within a specified sub-space. Obviously, better results can be obtained with larger calibration sets.

Another goal was to develop a technique that also compensates for the orientation error. A survey of existing tracker calibration methods was made to identify techniques that compensate for the rotational error. Only one technique was found [5], but it requires a lot of calibration points to be collected in order to work in contrast to the proposed method.

Yet another goal was to implement the technique in the form of a usable software library that can be integrated into third party products. Such implementation is currently available for downloading from the web site http://www.ncsa.uiuc.edu/VEG/VPS/emtc/. The code is written in $\mathrm{C}$ and compiled on an SGI workstation. Currently it has been integrated with the $\mathrm{dV} / \mathrm{MockUp} \mathrm{dV} / \mathrm{Immersion}$ (Division, Inc.) software as a sensor filter which is dynamically loaded into the sensor actor and executed each time the tracker data has been updated. A plan exists to integrate the software into WTK IDO (Sense8 Corporation) software.

\section{Future work}

Several issues need to be studied. First, a detailed study is required to identify a sub-volume near the tracking system that needs to be calibrated. In the present study, almost entire CAVE space was included; however, in reality there are places that are never reached by users. Excluding them from the calibration may lead to a better calibration quality of the remaining sub-volume. Currently, a study is going on to identify such places. Second, it needs to be studied how many measurements are enough to reach a specific level of the calibration quality. Third, the use of other basis functions for the fitting polynomials needs to be explored. In the present study, only the functions in the form $x^{p_{j}^{\prime}} y^{p_{j}^{\prime \prime}} z^{p_{j}^{\prime \prime}}$ were used. However, they may not be the best-suited ones taking into account the nature and the shape of the magnetic field distortions. Finally, the proposed method by itself does not remove the need for manual measurements in order to build a calibration table. Ideally, a tracking system should calibrate itself automatically 
as users work with the VR system. The following setup is currently under consideration: an accurate image-based tracking system runs in parallel with the electromagnetic tracking and from time to time provides a precise location and orientation for the calibration table while a user works with the VR system.

\section{Acknowledgments}

This work was performed at the National Center for Supercomputing Applications with a financial support from Caterpillar Inc. Several NCSA people helped to conduct the study: Henry Kaczmarski built a support device that was used to hold the tracker's receiver at the pre-defined locations, Lance Arsenault advised with physics/math issues. Additional information regarding the calibration of electromagnetic trackers was provided by Ascension Technology Corporation. Division Inc kindly shared the information needed to implement the sensor filter for $\mathrm{dV} / \mathrm{MockUp}$ sensor actor.

\section{References}

1. Raab F, Blood E, Steioner T, Jones H. Magnetic position and orientation tracking system. IEEE Transactions on Aerospace and Electronic Systems 1979. 15(5): 709718.

2. Nixon M, McCallum B, Frigh W, Price B. The effects of metals and interfering fields on electromagnetic trackers. Presence 1998. 7(2): 204-218.

3. Ghazisaedy M, Adamczyk D, Sandin D, Kenyon, DeFanti T. Ultrasonic calibration of a magnetic tracker in a virtual reality space. In: Proceedings IEEE Virtual Reality Annual International Symposium (VRAIS '95). Research Triangle Park NC, March 1995; 179-188.

4. Meyer K, Applewhite H, Biocca F. A survey of position trackers. Presence 1992. 1(2): 173-200.

5. Ellis S, Adelstein B, Baumeler S, Jense G, Jacoby R. Sensor spatial distortion, visual latency, and update rate effects on 3D tracking in virtual environments. In: Proceedings IEEE Virtual Reality (IEEE VR '99) Conference. Houston TX, March $1999 ; 218-221$.

6. Cruz-Neira C, Sandin D, DeFanti T. Virtual reality: the design and implementation of the CAVE. In: Proceedings SIGGRAPH '93 Computer Graphics Conference. ACM SIGGRAPH, August 1993; 135-142.

7. Kindratenko V, Kirsch B. Sharing virtual environments over a transatlantic ATM network in support of distant collaboration in vehicle design. In: Virtual Environments '98: Proceedings of the Eurographics Workshop. Göbel M, Landauer J, Lang U, Wapler M eds. Springer-Verlag. 1998.

8. Bryson S. Measurement and calibration of static distortion of position data from 3D trackers. In: Proceedings SPIE Conference on Stereoscopic Displays and Applications III. San Jose CA, February 1992; 244-255.

9. Czernuszenko M, Sandin D, DeFanti T. Line of sight method for tracker calibration in projection-based VR systems. In: Proceedings $2^{\text {nd }}$ International Immersive Projection Technology Workshop. Ames IO, May 1998. 
10. Kenwright D, Lane A. Interactive time-dependent particle tracing using tetrahedral decomposition. IEEE Transactions on Visualization and Computer Graphics 1996; 2(2): $120-129$.

\section{Figure legends}

Fig. 1. A surface built over the points from the validation table. True coordinates for the reference points are $\mathrm{A}(-3.45,5,-3.5), \mathrm{B}(3.51,5,-3.5), \mathrm{C}(3.51,5,3.5)$, and $\mathrm{D}(-3.45,5$, $3.5)$.

Fig. 2. Measured (a) and fitted (b) values of $x$ coordinate of the points from the validation table plotted against $x$ 's actual value. Plot a reveals some magnetic field distortions that are hard to see in Fig. 1: the value of $x$ measured from the right side of the transmitter is smaller than the actual one and is larger than the actual one when measuring it from the left side.

Fig. 3. Frequency histograms built for the distances between the true location of the receiver and the values reported by the tracker (a) and values after the fitting (b).

Fig. 4. Measured and fitted values of the azimuth for the points from the validation table. Each group of 8 consecutive points starting from the first point has the same true value of $x$, their true $z$ values run from -3.5 feet to +3.5 feet within each group. A similarity is observed in the azimuth changes within each group of points.

Fig. 5. A surface built over the points from the validation table after fitting the data with the $3^{\text {rd }}$ order polynomial. Four reference points are shown with their fitted coordinates, their true coordinates are the same as those in Fig. 1.

\section{Table legends}

Table 1. Summary of the electromagnetic trackers calibration techniques.

Table 2. Statistics derived from the validation data before and after fitting it by the $2^{\text {nd }}$, $3^{\text {rd }}, 4^{\text {th }}$, and $5^{\text {th }}$ order polynomials. 


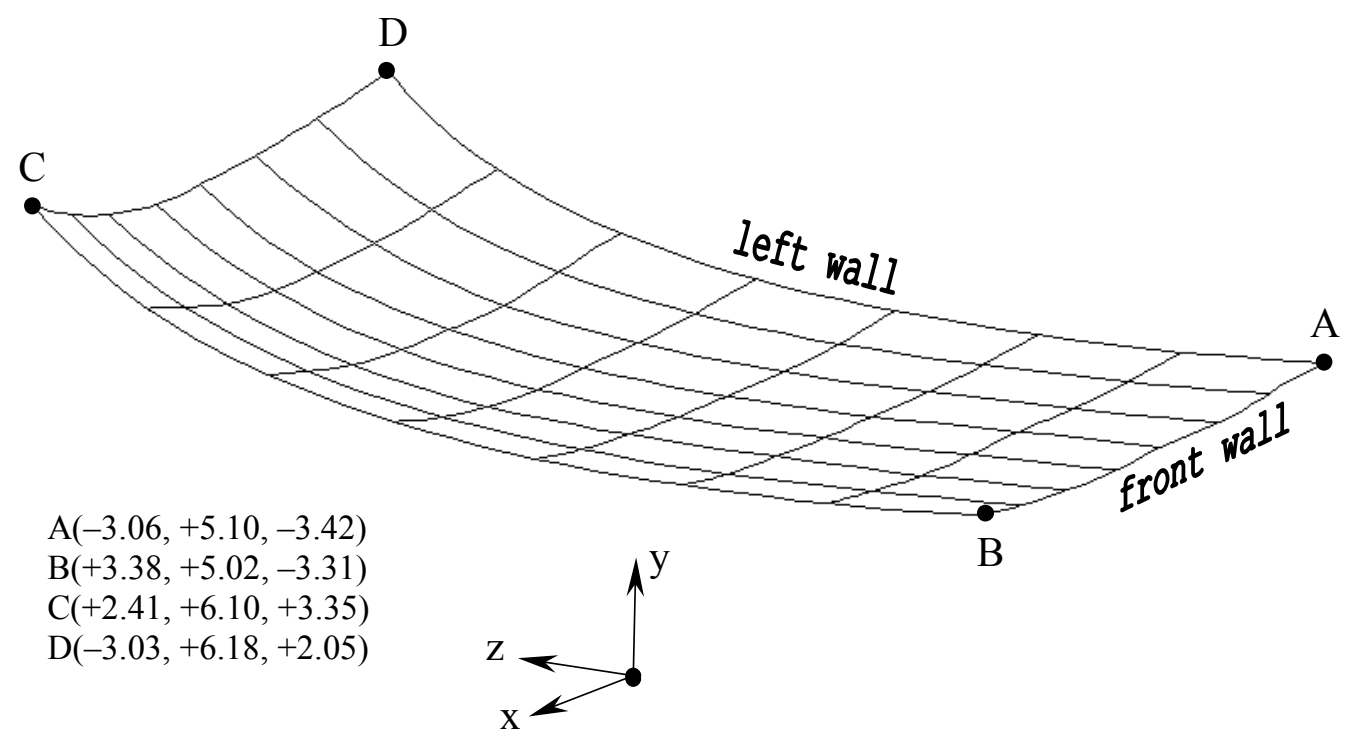

Fig. 1.
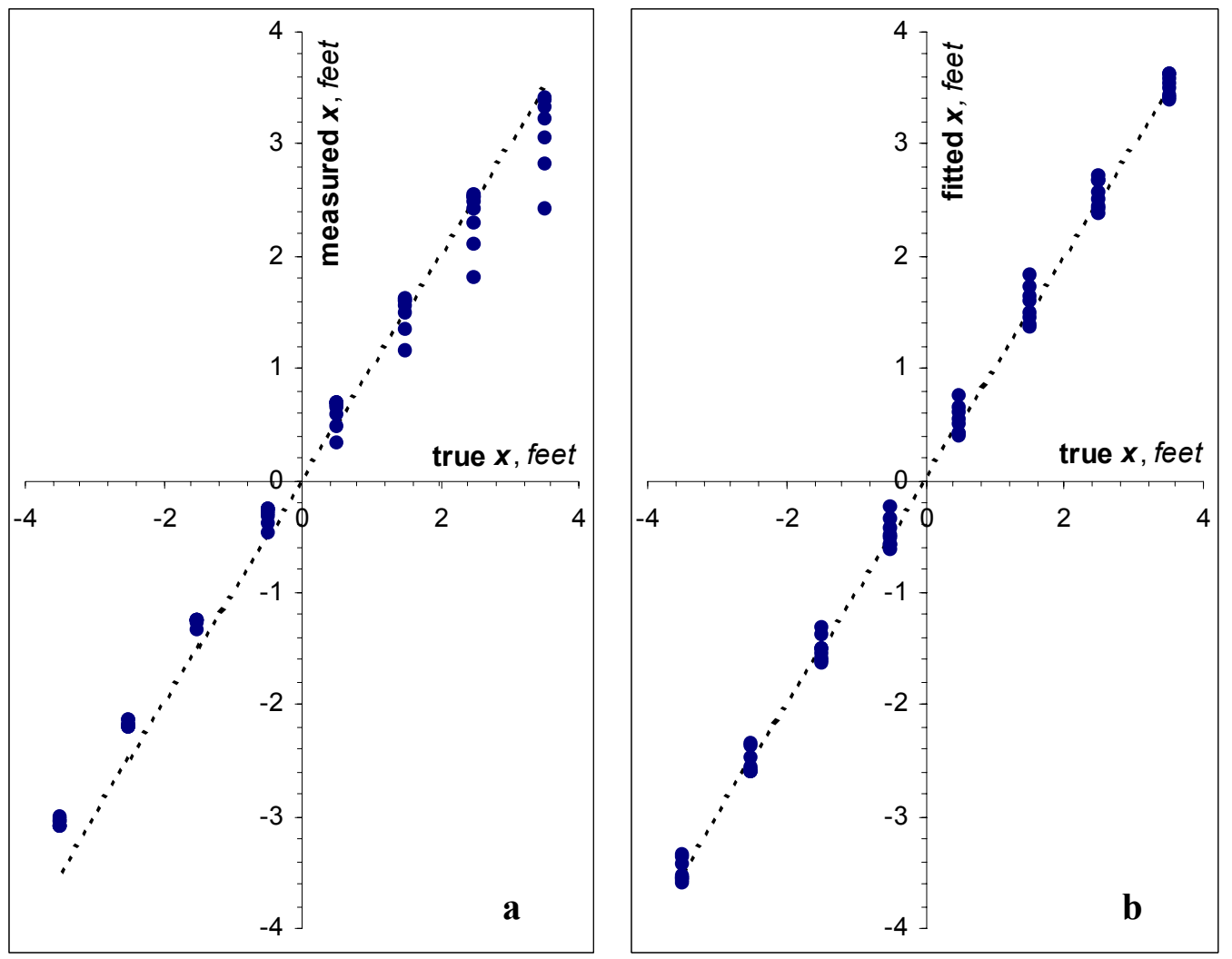

Fig. 2. 

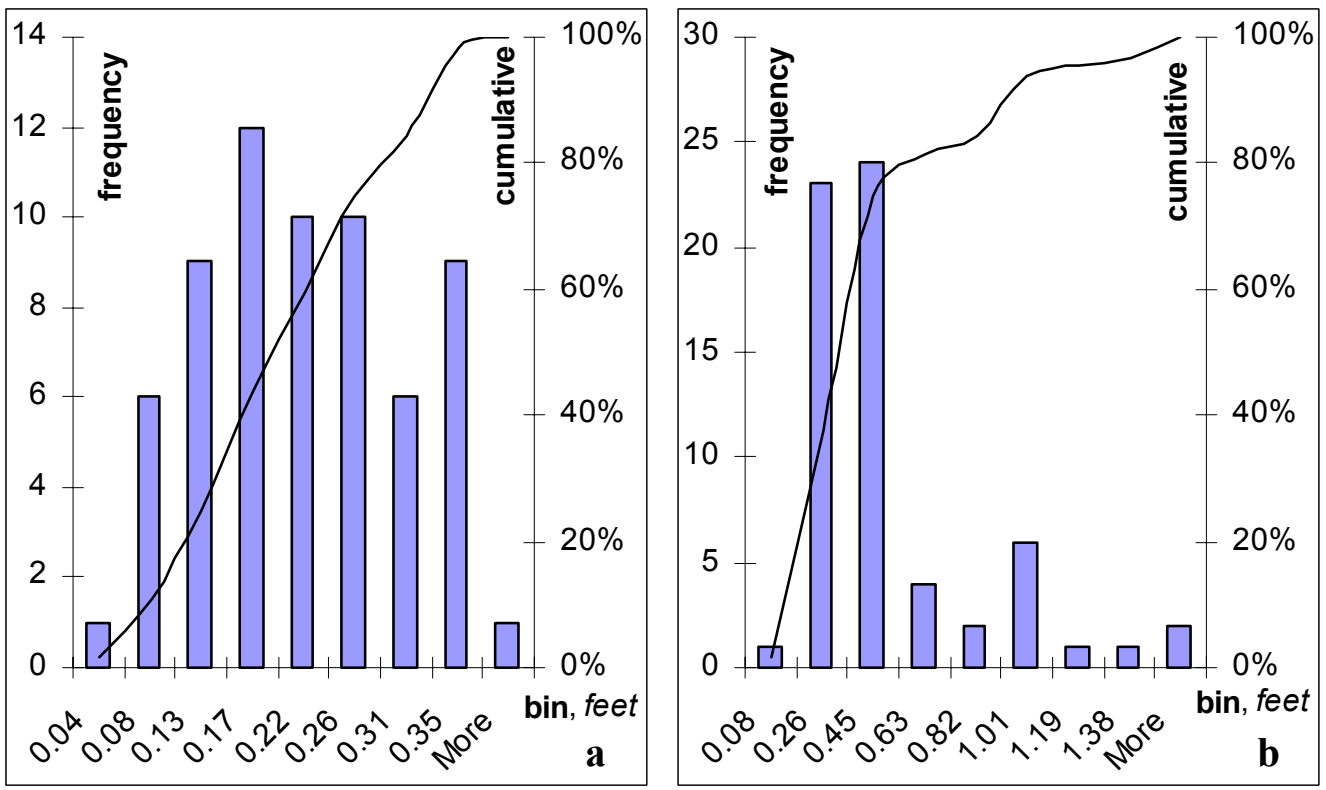

Fig. 3.

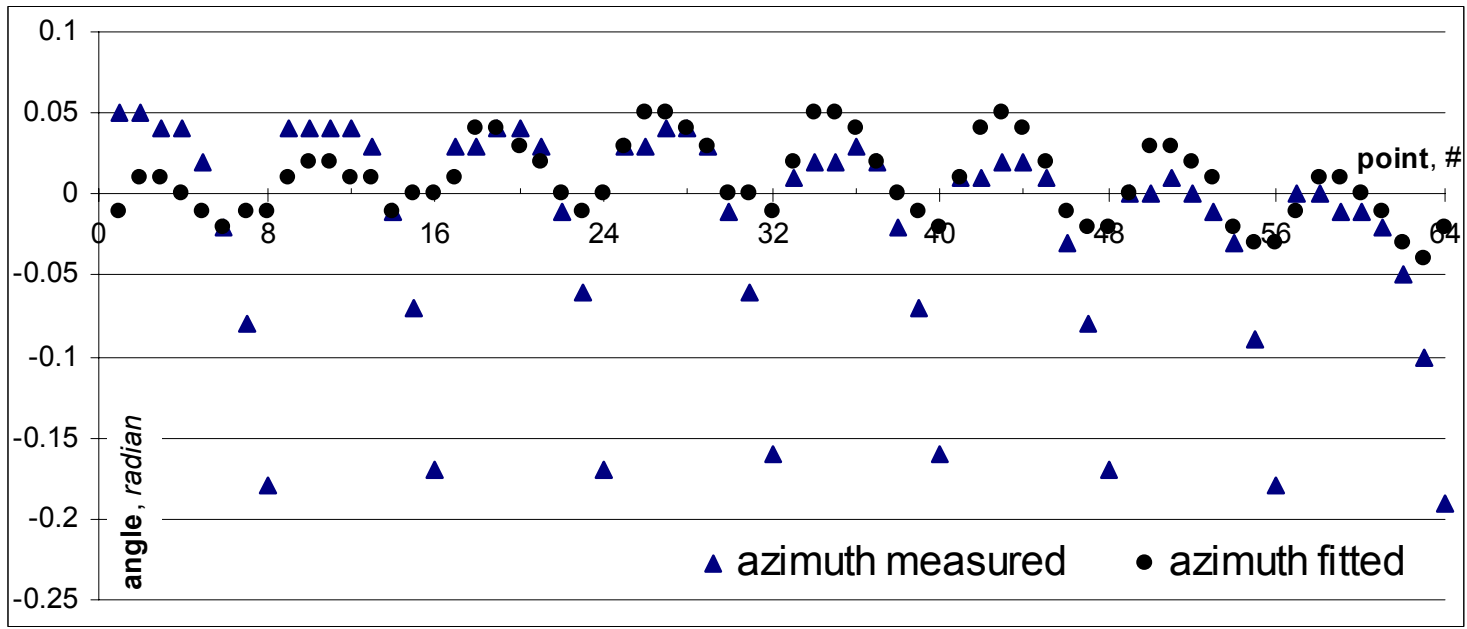

Fig. 4. 


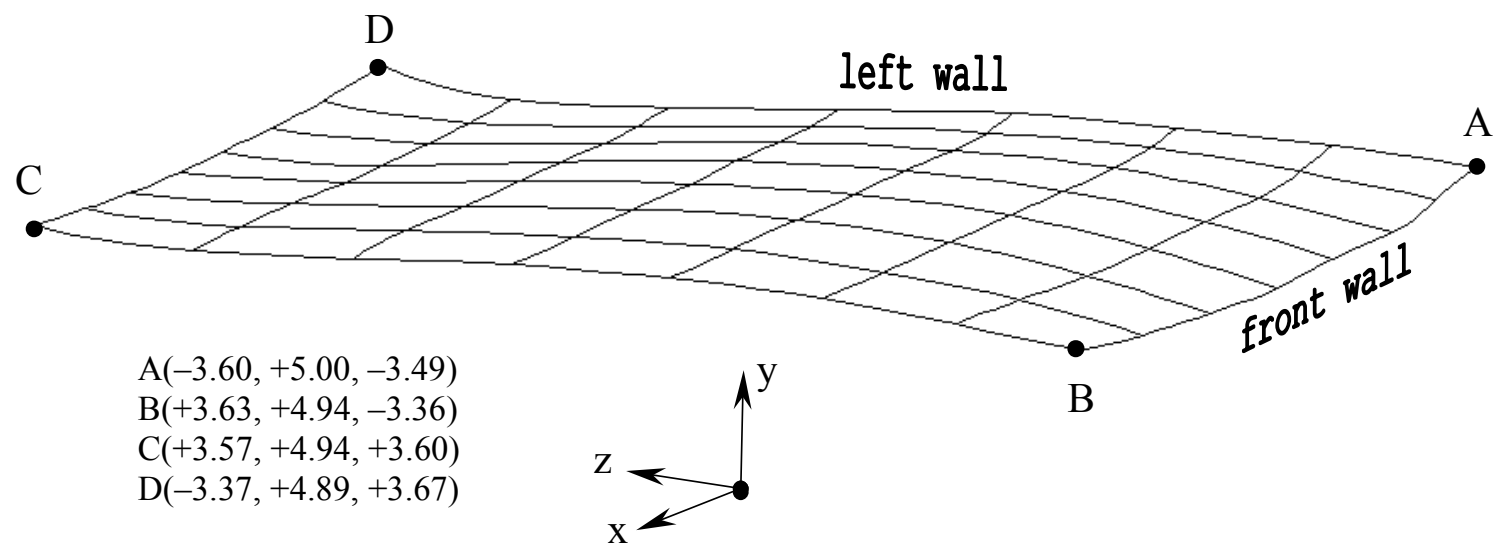

Fig. 5.

\begin{tabular}{|c|c|c|c|c|}
\hline Author(s) & $\begin{array}{l}\text { EMT Device / } \\
\text { VR system }\end{array}$ & Measurements & Position Correction & Orientation Correction \\
\hline S. Bryson & $\begin{array}{l}\text { Isotrack } \\
\text { / HMD }\end{array}$ & $\begin{array}{l}\text { 8'x'8x6' volume, } 12 " \\
\text { step, "measured vs. true" } \\
\text { calibration table }\end{array}$ & $\begin{array}{l}4^{\text {th }} \text { order polynomials, } \\
\text { weighted table lookup. } \\
\text { Twice reduced error }\end{array}$ & No \\
\hline $\begin{array}{l}\text { M. Ghazisaedy et } \\
\text { al. }\end{array}$ & $\begin{array}{l}\text { Flock of Birds } \\
\text { / CAVE }\end{array}$ & $\begin{array}{l}\sim 8 ' x 8 \text { 'x } 8 \text { ' volume, } 12 " \\
\text { step, "true vs. measured" } \\
\text { calibration table }\end{array}$ & $\begin{array}{l}\text { Trilinear interpolation. } \\
\text { Error reduction from } 4^{\prime} \\
\text { to } 0.27^{\prime}\end{array}$ & No \\
\hline $\begin{array}{l}\text { M. Czernuszenko } \\
\text { et al. }\end{array}$ & $\begin{array}{l}\text { Flock of Birds } \\
\text { / CAVE }\end{array}$ & $\begin{array}{l}\text { 6' from the floor, } \\
\text { "measured vs. true" } \\
\text { calibration table }\end{array}$ & $\begin{array}{l}\text { Trilinear interpolation. } \\
\text { Error reduction to } 0.2^{\prime}\end{array}$ & No \\
\hline S. Ellis et al. & $\begin{array}{l}\text { Fasttrack } \\
\text { / HMD }\end{array}$ & $\begin{array}{l}72 " x 48 " x 30 " \text { volume, } 6 " \\
\text { step, "measured vs. true" } \\
\text { calibration table }\end{array}$ & $\begin{array}{l}\text { Interpolation based on } \\
\text { tetrahedral elements. } \\
\text { Error reduced } 7 \text { times }\end{array}$ & $\begin{array}{l}\text { Quaterions rotation } \\
\text { No results published } \\
\text { on the error reduction }\end{array}$ \\
\hline $\begin{array}{l}\text { V. Kindratenko } \\
\text { (present work) }\end{array}$ & $\begin{array}{l}\text { Flock of Birds } \\
\text { / CAVE }\end{array}$ & $\begin{array}{l}\text { 8'x8'x6' volume, 2' step, } \\
\text { "measured vs. true" } \\
\text { calibration table }\end{array}$ & $\begin{array}{l}3^{\text {th }} \text { order polynomials } \\
\text { Error reduced } 4 \text { times }\end{array}$ & $\begin{array}{l}3^{\text {th }} \text { order polynomials } \\
\text { Error reduced } 3 \text { times }\end{array}$ \\
\hline
\end{tabular}

Table .1. 


\begin{tabular}{|c|c|c|c|c|c|c|}
\hline \multicolumn{2}{|c}{ variable } & \multirow{2}{*}{ non-fitted } & \multicolumn{4}{c|}{ fitted by the polynomial of the order } \\
\cline { 3 - 7 } & & 2 & 3 & 4 & 5 \\
\hline \multirow{2}{*}{$\Delta|x|$} & avr \pm std & $0.245 \pm 0.186$ & $0.296 \pm 0.165$ & $0.1 \pm 0.061$ & $0.129 \pm 0.092$ & $0.099 \pm 0.079$ \\
\cline { 2 - 7 } & $\min -\max$ & $0.02-1.10$ & $0.001-0.589$ & $0.003-0.306$ & $0.002-0.312$ & $0.001-0.282$ \\
\hline \multirow{2}{*}{$\Delta|y|$} & avr \pm std & $0.247 \pm 0.307$ & $0.111 \pm 0.062$ & $0.083 \pm 0.057$ & $0.109 \pm 0.056$ & $0.160 \pm 0.086$ \\
\cline { 2 - 7 } & $\min -\max$ & $0-1.18$ & $0.002-0.238$ & $0.001-0.226$ & $0.002-0.228$ & $0.014-0.324$ \\
\hline \multirow{2}{*}{$\Delta|z|$} & avr $\pm \operatorname{std}$ & $0.142 \pm 0.113$ & $0.129 \pm 0.095$ & $0.122 \pm 0.084$ & $0.086 \pm 0.074$ & $0.140 \pm 0.090$ \\
\cline { 2 - 7 } & $\min -\max$ & $0-0.650$ & $0.006-0.347$ & $0.002-0.292$ & $0.001-0.264$ & $0.000-0.456$ \\
\hline \multirow{2}{*}{$\Delta|d|$} & avr $\pm \operatorname{std}$ & $0.421 \pm 0.324$ & $0.368 \pm 0.145$ & $0.194 \pm 0.090$ & $0.207 \pm 0.100$ & $0.256 \pm 0.107$ \\
\cline { 2 - 7 } & $\min -\max$ & $0.078-1.563$ & $0.077-0.647$ & $0.036-0.395$ & $0.042-0.459$ & $0.062-0.468$ \\
\hline \multirow{2}{*}{$\Delta|\alpha|$} & avr $\pm \operatorname{std}$ & $0.048 \pm 0.053$ & $0.029 \pm 0.021$ & $0.020 \pm 0.015$ & $0.018 \pm 0.015$ & $0.034 \pm 0.023$ \\
\cline { 2 - 7 } & $\min -\max$ & $0.002-0.190$ & $0-0.08$ & $0-0.054$ & $0-0.055$ & $0.002-0.087$ \\
\hline \multirow{2}{*}{$\Delta|\beta|$} & avr $\pm \operatorname{std}$ & $0.038 \pm 0.041$ & $0.041 \pm 0.023$ & $0.029 \pm 0.018$ & $0.017 \pm 0.015$ & $0.049 \pm 0.038$ \\
\cline { 2 - 7 } & $\min -\max$ & $0-0.240$ & $0.001-0.085$ & $0.001-0.069$ & $0-0.073$ & $0.003-0.192$ \\
\hline \multirow{2}{*}{$\Delta|\gamma|$} & avr $\pm \operatorname{std}$ & $0.053 \pm 0.031$ & $0.028 \pm 0.026$ & $0.034 \pm 0.020$ & $0.033 \pm 0.023$ & $0.040 \pm 0.025$ \\
\cline { 2 - 7 } & $\min -\max$ & $0.005-0.163$ & $0.001-0.116$ & $0-0.086$ & $0.002-0.085$ & $0.002-0.088$ \\
\hline
\end{tabular}

Table .2. 